\title{
Convergence of LBS and AI
}

\author{
Sandeep Kumar, Mohammed Abdul Qadeer, and Sumit Kumar Singh
}

\begin{abstract}
Location Based Service (LBS) refers to class of applications that provides services based on the current or a known location. The location information can be obtained through the mobile communication network or the Global Navigation Satellite Systems (GNSS) [8]. But in all the cases after extracting information we have to make an exhaustive processing over this extracted geographic information. And this exhaustive processing requires a significant amount of time if it is performed by traditional techniques.

Artificial Intelligence, on the other hand, has been proven to be useful in the interpretation of spatial resource information. On of the artificial intelligence technique to support spatial processing is artificial neural networks (ANNs). The recent developments in ANN technology have made it more ofan applied mathematical technique that has some similarities to the human brain.

The integration of LBS with AIoffers a potential mechanismto lower the analysis-time of spatial information. One major advantage is that this integration allows the interpretive result from a small area to be transferred to a larger, geographically similar area.

The objective of this paper is to discuss about the relativity of LBS services and Artificial Intelligence technologies and to uncover the benefits of converging LBS and AI.
\end{abstract}

Index Terms_LBS, GIS, AI, ANNs.

\section{LOCATION BASED SERVICES AND THEIR APPLICATIONS}

\section{A. Location Based Services (LBS)}

Location Based Services (LBSs) [16] provide a mean to service the user request (or to provide the information to user) depending upon the proximity of the user. The proximity of user can be determined by using Global Navigation Satellite System (GNSS), Geographic Information System (GIS) and Wireless Communication (WC) technologies.

LBS offer modern world the tool and technologies for efficient management, continuous control and comfortable management. More and more people involve LBS in their industry and day to day life to better achieve their goals.

It employs accurate, real-time positioning to connect users to points of interest and advises them of the current conditions such as traffic and weather conditions, or provides routing and tracking information using wireless

Manuscript received August 9, revised September 7, 2012. This work was supported in part by Aligarh Muslim University.

S. Kumar is with the Tata Consultancy Services Limited, Pune India (email: sandeepkumar@zhcet.ac.in).

M. A. Qadeer is with the Aligarh Muslim University. Uttar Pradesh, Aligarh, India (e-mail: maqadeer@gmail.com).

S. K. Singh is with the Tata Consultancy Services Limited, Pune India (e-mail: sumitkumarsingh@zhcet.ac.in). devices. It is important to integrate the mobile computing technologyand the GIS technology in order to meet the needs of LBS, which is considered one of the most promising applications of GIS[10][13].

\section{B. Applications of LBS Services}

LBS is a concept that can be utilized widely. LBS can be applied in public and safety industry, such as emergency service in medical [9]; tracking industry[11], such as fleet management; personalization information industry, such as query the nearest restaurant; navigation industry, such as digital map; payment and so on. It can be particularlypowerful when combined with other user profile information to offer personalized and location sensitive responses to customer; this form is called the context aware system. Following figure shows the different application areas of LBS Services:

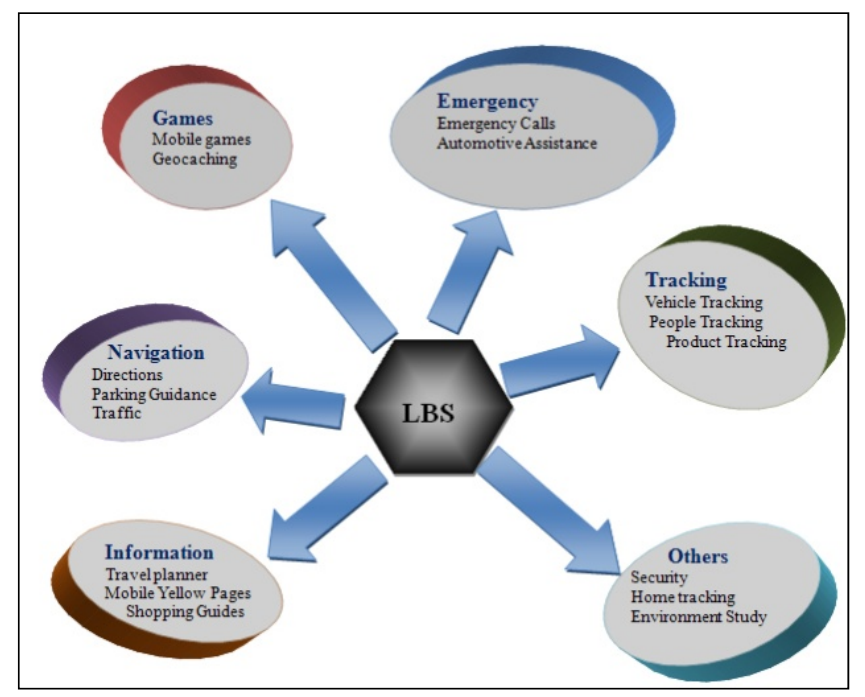

Fig. 1. Application areas of LBS services

\section{ARTIFICIAL INTELLIGENCE AND ITS SUBFIELDS}

There exists a number of conflicting definitions of AI. One particularly useful definition, nevertheless, is provided by Smith in reference [1]: "Artificial intelligence may be regarded as an attempt to understand the processes of perception and reasoning that underlie successful problemsolving and to incorporate the results of this research in effective computer programs," However, this definition does not answer purpose of current technological development of Artificial Intelligence in location based services.

Time being, we can consider the AI as a collection of powerful and rigorous programming techniques studying the nature of intelligence by building computer systems, and the application of these concepts in solving real-world problems. From this point of view we can say AI is seeking 
develop systems that attempt to mimic human intelligence without claiming an understanding of the underlying processes involved, means it is abstracting the underlying information. Further to this, Artificial intelligence (AI) is a branch of computer science that aims to simulate the thought process of the human brain, commonly through the use of software [3]. AI is the fundamental technology for building expert systems. In artificial intelligence, the scientists study reasoning and machines, and consider such questions as whether a machine can properly be considered to reason orthink, and what would count as a test for reasoning (called its performance measure).

\section{A. Subfields of AI}

The growth in the areas of AI has been increased significantly from the last decade. At present AI has following main branches of research (new technologies are being developed constantly):

1) Artificial neural networks (ANNs)- simulate the working ofneurons in the brain,

2) Natural language processing (NLP) - aims to produce computersystems that can understand, translate and communicate inhuman languages,

3) Genetic Algorithms - solve problems by a loose analogywith biological evolution by natural selection,

4) Knowledge based systems - encode human expertknowledge in such a way a computer can reason with it,

5) Case-based reasoning - simulates how humans reasonfrom past experience,

6) Robotics - focuses on the construction of intelligentrobots that adapt to their environment,

7) Vision - focuses on tasks such as face recognition.

Almost all the above mentioned technologies can make a LBS service more sophisticated e.g.

- Using the NLP related tools the LBS becomes more interactive , moreover physically disabled (like blind) can also use the service

- Using Genetic Algorithm more complex spatial information processing can be performed within permissible amount of time with accuracy and rationally.

- Using Knowledge based system it will be easy to guide the touristy effectively.

- Case based reasoning can utilize the experience factor to design a tour.

- Robotics is the key technique to make the real time location based services (RTLBS) possible.

- Using vision and related processing the LBS service can guide the user more effectively.

- And at last but the most important technique, ANN is the main contributing and promising technology from AI area to LBS era. This is because in GIS(Geographical Information System) ANN has always been used in some form. So, in this paper our main concentration will revolve around ANNs.

\section{PRELIMinaries}

LBS based system are generally very complex because they uses the GIS system. These systems require sophisticated reasoning mechanism because these systems have to deal with spatial information and databases. However, spatial information databases usage is limited, especially for the systems where data are verydynamic and need intelligent decision making support in thepresence of some uncertainty. In [5][17][12][6] ithas been suggested that neural networks and case-basedreasoning $\mathrm{n}$ be used for modeling ecological systems.

A typical LBS system is a combination of GIS and ANNs. In such an application GIS is used to develop spatial, predictor drivers and perform spatial analysis on the results. On the other hand AI is used to learn the patterns of development in the region and test the predictive capacity of the model. An agent-based distributed artificial intelligent GIS paradigm is discussed by Wang [7]. He provided Geoletbased DGIS prototype system to fully accommodate distributed, heterogeneous and speedy development in dynamic computing environments.

\section{RELATED WORK}

Convergence of artificial intelligence with LBS in not new, from several years there is a remarkable convergence of AI and GIS (Geographical information System). And the LBS in nothing but is an application of GIS. In fact AI and GIS are mutually supportive technologies.On one end GIS provides a wide range of applications where we can use the technologies from AI era, while on the other hand AI provide excellent tools for implementing GIS based projects which requires high level of decision making and reasoning and LBS is one such application.

Other factor that supports the convergence of LBS and AI is the mathematical model of AI (especially neural network) and LBS.

\section{Neural Network, Fuzzy Logic and LBS Services}

As discussed in [2] the two main properties required by almost all GIS based application is classification and feature extraction. In [2] discussion also has been made for implementing these properties using the techniques of Artificial Neural Networks. In [1] the discussion has been made for demonstrating the usefulness of ANNs in modeling spatial interaction and classifying spatial data. In [4] a digital cartography has been discussed which was mainly based on recognition of optical character using artificial neural network.A number of spatial processing applications based on fuzzy logic also have been discussed in different literature. Apart from these simple projects, [15] [19] discusses the land suitability analysis and land transformation analysis using the spatial processing of GIS and powerful reasoning of AI and ANNs.

In [18] a simple LBS system has been discussed devoted to travel forecasting and transport planning in Boston metropolitan area. The LBS system was designed to quantify trips within the urban area through the representation of the land usetransportation system interaction.

In [14] a detail discussion over established parametric methods (multiple regression and discriminant function analysis) and artificial intelligence techniques (neural networks and case-based reasoning) for use within a GIS 
has been made. Predictive model[25][24] was a case-based system relying on statistical parametric tests, neural network rule extraction and expert advice/literature to justify its indexing and weighting functions. With information from all these techniques, expert knowledge use to determine the most appropriate level and form of integration.

One other interesting GI application using ANNs was discussed by Bares in [21]. In this paper AI and GIS are combined to handle the existing non-linear relation between the target variable (frost days) and the predictors (location and elevation of the meteorological stations).

Al-Bastaki [23] utilized the concepts of neural network and generic algorithms for compressing the GIS images and also explained the algorithms for restoration from compression(Fig. 1). His observations show that compressibility of AI-based methods and the image distortion depends on the number of clusters used in adaptive neural network process. He also showed that the adaptive neural network algorithms appear to give extremely good performance for GIS image compression.

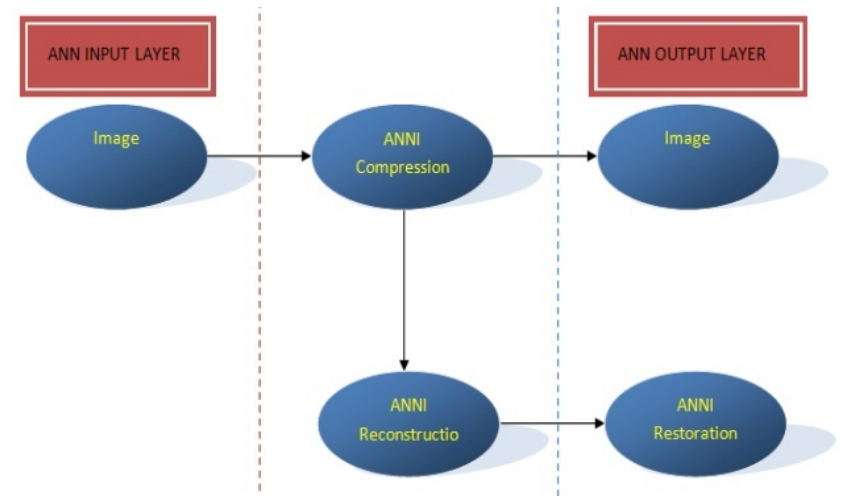

Fig. 2 Block diagram of artificial neural network used by Al-Bastaki [23] for compression and restoration of GIS image datasets.

\section{AI IN LBS}

As discussed in Section-I LBS services are a special class of GIS based services that provide the user-services (information) depending upon the current location of the user. Following figure shows a typical structure of an LBS service:

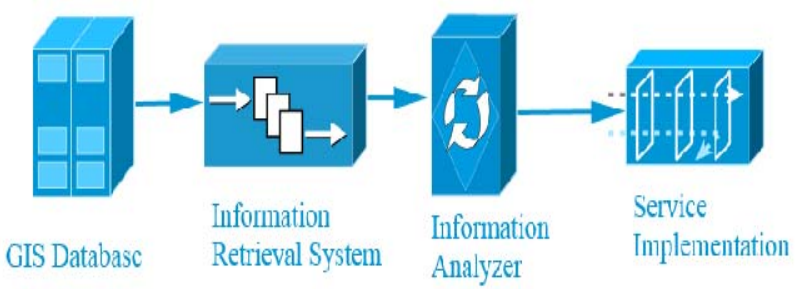

Fig. 3. Typical structure of a LBS system.

The first part i.e. GIS Database is devoted to store all needed geographical information.

The second part i.e. Information Retrieval system is the part that access the GIS database to fetch the needed geographical information

The third part i.e. Information Analyzer is the decision taking part of the LBS services and is devoted to analyze the retrieved information for determining the user specific information
The last part i.e. Service Implementerprovide the service to user as decided by the Information Analyzer part. It is simply an application, so we will not discuss about this in this paper.

The first part is purely based on GIS technology. The second part can be implemented either using traditional GIS retrieval system or it can be implemented using more efficient and modern AI based retrieval systems.

Third part is initially was based on pure traditional GIS technologies, now moving towards more featured ANNs technology. ANNs provide an excellent support for selecting approaches in spatial patterns which is also somewhat more efficient with respect to processing time as discussed in[21].

Further to this, ANNs can be used for predicting accuracy and appropriateness of spatial modeling technique. Moreover, other AI based processing techniques can also be used to gain insight into importantspatial functions and processes through rule extraction andfactor sensitivity tests.

\section{RELATIONSHIP BETWEEN LBS WITH AI}

In LBS what we require is that we have to analyze the spatial information for a particular pattern and extraction of features from these spatial images. Depending upon the analysis results and extracted features decision device takes the designated decision.There exists a number of ways for analysis on such method is based on threshold model discussed in [20]. But as stated in [20] this model of analysis is rigid as this model matches the spatial images with standard defined images and then determines the closeness with a particular situation according to the degree of matching. So, it seems to be inefficient as well as time consuming process. Moreover the decision making is also not rational.

But now, after the evolution and advancements in AI we can analyze the spatial information more effectively and efficiently as compared to our threshold model. Decision support systems, built to help humans make informed decisions, are classic examples of where GIS can be effectively coupled with reasoning techniques developed in the AI community. AImethods have potential for better implementation of prediction of the spatial variables distribution which is required frequently in the LBS based system.

\section{CONCLUSION}

In this paper we have seen that the convergence of AI and LBS is not new. But still we require some more tools and technologies to converge both these fields. First thing that we must have is the transferring of information from GIS based system to AI based system and vice versa. And at last, we conclude that the convergence of AI and LBS open a new era of technologies.

\section{REFERENCES}

[1] S. Openshaw and Ch. Openshaw, "Artificial Intelligence in Geography,” Chichester, Wiley \& Sons, 1997.

[2] B. G. Lees and K. Ritman, "Decision tree and rule induction approach to integration of remotely sensed and GIS data in mapping vegetation 
in disturbed or hilly environments," Environmental Management, vol. 15, pp. 823-831, 1991.

[3] L. M. Cowardin, P. M. Arnold, T. L. Shaffer, H. R. Pywell, and L. D. Miller, "Artificial intelligence and natural resource management," Science, 237, pp. 262-267, 1988.

[4] V. Voženílek, "Cartography for GIS - geovisualization and map communication,” Olomouc, Univerzita Palackého v Olomouci.

[5] C. J. A. Bradshaw, M. Purvis, R. Raynov, Q. Zhou and L. S. Davis, "Predicting patterns in spatial ecology using neural networks: modeling colonization,"New Zealand fur seals, in Proc. of the International Symposium on Environmental Software Systems (ISESS) 1999.

[6] M. H. Hassoun, "Fundamentals of Artificial Neural Network," Cambridge, Massachusetts Institute of Technology (MIT) Press, 1995

[7] Y. Wang, C. Rizos, L. Ge, M. Anner and M. Dwyer, "Distributed artificial intelligence in GIS - geolet: A distributed geo-spatial information retriever," Journal of spatial science, vol. 50, no. 2, pp. 37- 50, 2005.

[8] Q Ren, M. H. Dunham, "Using Semantic Caching to -Manage Location Dependent Data in Mobile Computing," In the 6th Annual International Conference on Mobile Computing and Networking, Boston: ACM Press, 2000, pp.210-222

[9] Jinsoo Ahn, Jungil Heo, Suyoung Lim, Jungho Seo, Wooshik Kim, "A Study of Healthcare System for Patient Location Data Based on LBS,"

[10] Balqies Sadoun and Omar Al-Bayari, "LBS and GIS Technology Combination and Applications," in Proceeding International Conference on Computer Systems and Applications, 2007.

[11] N. Marmasse and C. Schmandt, "Safe \& sound - a wireless leash," in Proceedings of CHI 2003, extended abstracts. 2003. pp. 726-727.

[12] S. L. Ozesmi and U. Ozesmi, "An artificial neural network approach to spatial habitat modelling with interspecific interaction,” Ecological Modelling, vol. 116, pp. 15-31, 1999.

[13] Balqies Sadoun and Omar Al-Bayari, "LBS and GIS Technology Combination and Applications", in proc. International Conference on Computer Systems and Applications, 2007

[14] K. Macgillivray, J. B. Wilson and A. Holt, "The Use of Artificial Intelligence Techniques and GIS for Predictive Vegetation Modelling," in Proc. of the $11^{\text {th }}$ Annual Colloquium of the Spatial Information Research Centre (SIRC 99), Dunedin, New Zealand, December $13-15^{\text {th }}, 1999$.

[15] F. Wang, "The use of artificial neural networks in a geographical information system for agricultural land-suitability assessment," Environment and Planning, vol. 26, pp. 265-284, 1994.

[16] Christian S. Jensen, Anders F. Christensen, Torben B. Pedersen. "Location-Based Services-A Database Perspective," pdf, pp.1-10

[17] T. Chon, Y. S. Park, K. H. Moon and E. Y. Cha, "Patternizing communities by using an artificial neural network," Ecological Modelling, vol. 90, pp. 69-78, 1996.

[18] A. Dantas, K. Yamamoto, M. V. Lamar and Y. Yamashita, "Neural Network for Travel Demand Forecast Using GIS and Remote Sensing," in Proc. of International Joint Conference on Neural Networks (IJCNN'O0), vol. 4, 2000.

[19] B. C. Pijanowski, D. G. Brown, B. A. Shellito and G. A. Manik, "Using neural networks and GIS to forecast land use changes: a LandTransformation Model," Computers, Environment and Urban Systems,vol. 26, no. 6, pp. 553-575, 2002.

[20] V. Voženílek, "Time and Space in Network Data Structures for Hydrological Modelling," in M. Craglia and H. Onsrud: Geographic Information Research - Trans-Atlantic Perspectives. London, Taylor \& Francis, pp. 189-202, 1999.
[21] S. Mann and G. L. Benwell, "The integration of ecological, neural and spatial modeling for monitoring and prediction for semi-arid landscapes," Computers and Geosciences, vol. 22, no. 9, pp. 10031012, 1996.

[22] M. Beres, L. Foresti, R. Tapia and M. Kanevski, "Frost risk mapping using neural networks and GIS decision models," Geophysical Research Abstract, 10, 2008.

[23] Y. A. L. Al-Bastaki, "GIS Image Compression and Restoration: A Neural Network Approach,” Information Technology Journal, vol. 5, no. 1, pp. 88-93, 2006.

[24] O. Nelles, "Nonlinear System Identification," Berlin, Springer-Verlag, 2000.

[25] S. McKillup and Statistics Explained, "An Introductory Guide for Life Scientists,” Cambridge, Cambridge University Press, 2006.

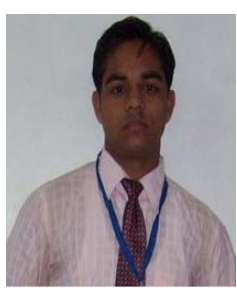

Sandeep Kumar is a Software developer with Tata Consultancy services limited, working with Microsoft client for Online services development. He has 14 research publications and has attended various national/international conferences. The main area of interest included Artificial Intelligence, Neural Network and Application of computational techniques and programming in solving / analyzing biological problems / phenomenon.

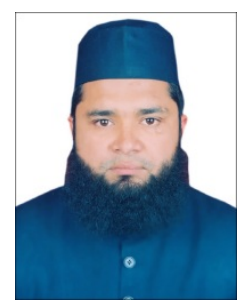

Mohammed A Qadeer (MIEEE, LMIETE, LMCSI) is an Asst. Professor with the Department of Computer Engineering, Aligarh Muslim University, India. Earlier, he was working with Cisco Systems Inc. as a Network Consulting Engineer with the Advanced Services division in the APAC region. He received his B.Sc. Engineering (Computer Engineering) from Aligarh Muslim University in1996. He has an experience of 15 years in the area of computer networks and systems. He served as a Technical Co-Chair for IEEE WOCN 2012, 2011, 2010, Technical CoChair IEEE AH-ICI 2012, 2011, International Steering Committee for ICACT2012, 2011, 2010 and as TPC member for CCNC 2011, 2010, INMIC 2009, AH-ICI 2009, WIA 2009 and MMA2009. He has been session chair and TPC reviewer for many IEEE/ ACM conferences and is a reviewer for IET Communications Journal as well. He is on the editorial board of International Journal of Digital Multimedia Broadcasting. Established global and nationwide setups of Internet Service Providers (ISP), Internet Exchange Points (IXP), Internet Data Centre (IDC) and Content Delivery Networks (CDN) both from a Networks and Systems perspective. His areas of research are computer networks, wireless networks, mobile computing, next generation networks, IMS, LTE, WiMAX, 4G, WiBro etc.

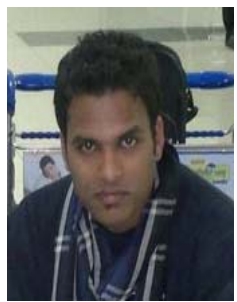

Sumit Kumar Singh is with Tata Consultancy Services Limited. He is highly enthusiastic to use IT for management. 